\title{
An experimental study of permeability determination in the lab
}

\author{
E. Lock, M. Ghasemi, M. Mostofi \& V. Rasouli \\ Department of Petroleum Engineering, Curtin University, Australia
}

\begin{abstract}
Understanding the flow characteristics in laminar and turbulent flow regime is important for different aspects of reservoir and production engineering. One of the most important parameters in fluid flow is the permeability of the porous media. It is common practice in the industry to use Darcy and Forchheimers equations for characterising the fluid flow in the porous media at laminar and turbulent regimes, respectively.

Core flooding experiments were performed with 1.5-inch diameter size core samples from limestone and sandstone formations. The permeability of the samples was measured in the laminar regime at basis flow rate. Then, the flow rate was increased in different steps and permeability was measured, accordingly. The plot of permeability versus flow rate was used to track the evolution of the flow regimes in the core porous media.

There are different challenges to monitor the transition between laminar and turbulent regime through core flooding experiments. These challenges are discussed in this paper through both literature review and also experimental results. The results indicated that the core sample preparation, experiment control parameters and also test profiles are important aspects when measuring permeability in the lab.

Keywords: permeability, laminar, turbulent, Darcy law, pressure drop.
\end{abstract}

\section{Introduction}

Permeability is a fundamental reservoir property and is extremely important for the reservoir engineers to determine during exploration of a well. Testing under laboratory conditions are tough and requires vigilance and control of many aspects. As a result of this, the permeability needs to be measured at different 
conditions when variables are changed to obtain an understanding how it changes due to change of different parameters. It is also important to determine the permeability in the laminar regime as the turbulent regime can create problems around the wellbore. Therefore it is necessary to find the boundaries between the two flow regimes by performing the core flooding experiments.

The fundamental law of fluid motion in porous media is Darcy's Law. The mathematical expression developed in 1856 states that the velocity of a homogenous fluid in a porous medium is proportional to the pressure gradient and inversely proportional to the fluid viscosity (Tarek and McKinney [1]). Mathematically this can be expressed as:

$$
q=-\frac{k A}{\mu} \frac{d p}{d L}
$$

where $q$ is the flow rate in cc/min and $A$ is the cross section area of flow in $\mathrm{cm}^{2}$. In this equation, $\mu$ and $d p / d L$ are fluid viscosity in $\mathrm{cp}$ and gradient of pressure loss in atm/cm, respectively. In Equation (1), the fluid is considered neutral imposing no chemical reaction to the porous media and also incompressible. The fluid flow is assumed single flow and liquid, which is subjected to laminar flow.

Following laminar flow assumption, the permeability is considered as flow rate independent since the pressure drop in the laminar regime is linearly function of flow rate. However, the flow pattern eventually converts to turbulent regime as the flow rate increase and as a result would not obey Darcy equation.

An expanded form of Darcy law was developed in 1901 by Phillip Forchheimer and named Forchheimers law (Barree and Conway [2]). He discovered that as the flow velocity increases, the inertial effects start dominating the flow. As a result of this, he developed an equation which is widely used to determine permeability in the turbulent regime as is shown below. An inertial coefficient $\beta$ is defined with a velocity squared term introduced. The non-Darcy flow coefficient is negligible at low flow velocities and is generally omitted from liquid flow equations (Macini et al. [3]):

$$
-\frac{d P}{d L}=\frac{\mu}{k} v+\beta \rho v^{2}
$$

where, $v$ is velocity in $\mathrm{m} / \mathrm{s}$ and $\rho$ is fluid density in $\mathrm{kg} / \mathrm{m}^{3}$. In this equation, $\beta$ is the non-Darcy flow coefficient or inertial coefficient is $\mathrm{m}^{-1}$.

Determining the boundary between laminar and turbulent regimes is subjected to several challenges and special care should be employed. After passing this boundary, characterising the turbulent regime is also a difficult task and rigours test procedure should be applied to make sure that during these experiments, the porous media property is not changed.

In this paper the challenges involved when testing the permeability at different flow rates will be examined to a limited extent through literature review and some laboratory experiments. 


\section{Experimental program}

As mentioned, in this paper, some challenges for determining the transition between turbulent and laminar regimes are introduced. These measurements were performed through linear flooding experiments with hydrostatic core flooding setup. A schematic of this setup is illustrated in Figure 3. In the following subsections the sample preparation and the core flooding test procedure will be discussed.

\subsection{Sample preparation}

The experiments were performed on both sandstone and limestone samples. The samples were all cleaned using the Dean-Stark toluene apparatus shown in Figure 1 and left overnight to dissolve any oil and other fluids. All samples were then dried in an oven overnight to evaporate any remaining toluene.

All samples then underwent routine core analysis using unsteady-state Permeameter as shown in Figure 1. This was to estimate the permeability of the samples using nitrogen as the flowing fluid before doing the experiment. The summary of these measurements are given in Table 1 .
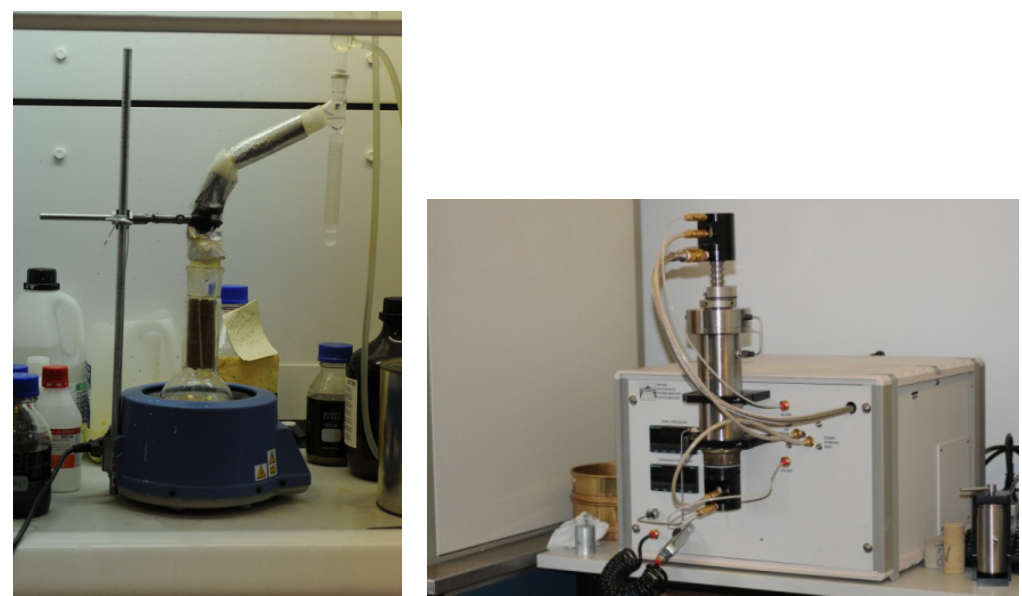

Figure 1: Dean-Stark apparatus (left) and Permeameter (right).

Table 1: $\quad$ Properties of core samples.

\begin{tabular}{|c|c|c|c|c|c|c|}
\hline Sample & $\begin{array}{c}\text { P conf } \\
\text { (psi) }\end{array}$ & $\begin{array}{c}\text { Viscosity } \\
(\mathbf{c P})\end{array}$ & $\begin{array}{c}\text { Diameter } \\
\text { (cm) }\end{array}$ & $\begin{array}{c}\text { Length } \\
\text { (cm) }\end{array}$ & $\begin{array}{c}\text { Area } \\
\mathbf{( c m}^{\mathbf{2}}\end{array}$ & $\begin{array}{c}\text { Permeability } \\
(\mathbf{m D})\end{array}$ \\
\hline $\mathrm{D}-01$ & 522.6 & 1 & 3.8 & 7.06 & 11.4 & 11.48 \\
\hline $\mathrm{D}-02$ & 507 & 1 & 3.68 & 5.73 & 10.6 & 20.89 \\
\hline $\mathrm{T}-01$ & 500 & 1 & 3.81 & 5.0 & 1.14 & 20.0 \\
\hline $\mathrm{C}-01$ & 517.6 & 1 & 3.77 & 9.48 & 11.2 & 3.0 \\
\hline
\end{tabular}




\subsection{Core flooding test}

In the setup, pressure transducers are located at both ends of the core flooding to measure the inlet and outlet pressure. The data is transferred to the computer and graphed against time. When the pressure is stabilized and showing little variation on the graph, the pressure is used to calculate the permeability.

The samples were saturated at different differential pressure to investigate the challenges of the flooding experiments under the influence of this parameter. Example of this effect will be illustrated in the next section.

The experiments were performed at different flow rates in order to pass laminar regime and enter the turbulent regime. The difference between overburden pressure and the mean pressure i.e. average of inlet and outlet pressure was kept constant to avoid the effect of overburden pressure on the permeability measurement.

The flow rates were selected according to evolution of the permeability in order to gain more insights about the boundary of the laminar and turbulent regimes. The base flow rate in which the permeability is measured was small enough to be in laminar flow rate. Tables 2 and 3 display the test data obtained for two tested samples (D-01 and D-02), respectively. More information about the flow rate selection is given in the next section.

Table 2: $\quad$ D-01 sample test data results.

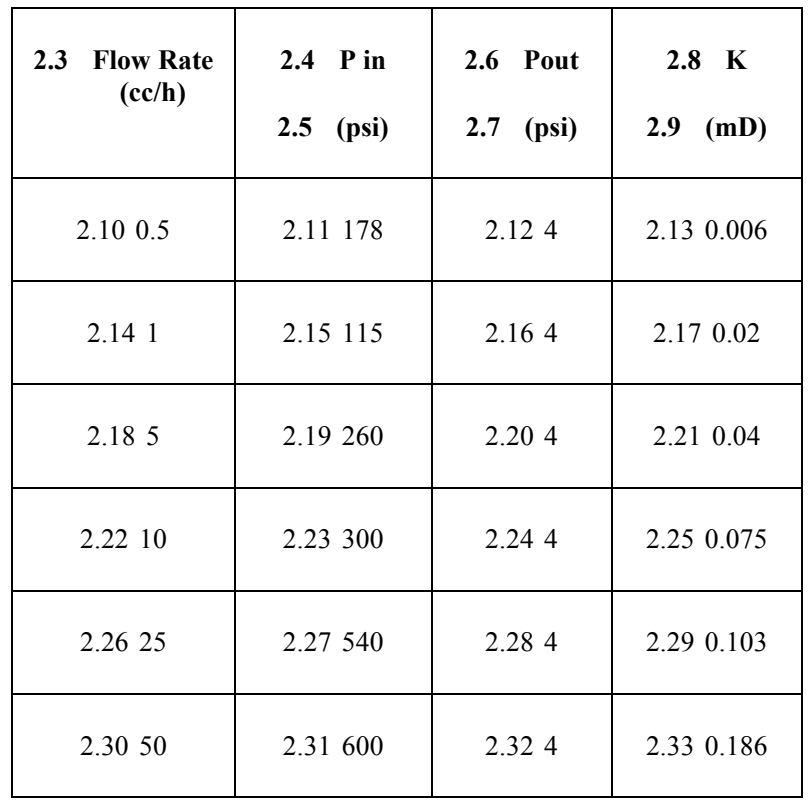


Table 3: D-02 sample test data results.

\begin{tabular}{|c|c|c|c|}
\hline $\begin{array}{c}\text { Flow Rate } \\
(\mathbf{c c} / \mathbf{h})\end{array}$ & $\begin{array}{c}\text { P in } \\
\text { (psi) }\end{array}$ & $\begin{array}{c}\text { P out } \\
\text { (psi) }\end{array}$ & $\begin{array}{c}\text { K } \\
\text { (mD) }\end{array}$ \\
\hline 1 & 65 & 4 & 0.03 \\
\hline 5 & 40 & 4 & 0.27 \\
\hline 10 & 36 & 4 & 0.603 \\
\hline 30 & 54 & 4 & 1.16 \\
\hline 50 & 89 & 4 & 1.14 \\
\hline 70 & 125 & 4 & 1.12 \\
\hline 80 & 145 & 4 & 1.09 \\
\hline 100 & 173 & 4 & 1.14 \\
\hline 120 & 195 & 4 & 1.21 \\
\hline 150 & 228 & 4 & 1.29 \\
\hline 200 & 282 & 4 & 1.39 \\
\hline
\end{tabular}

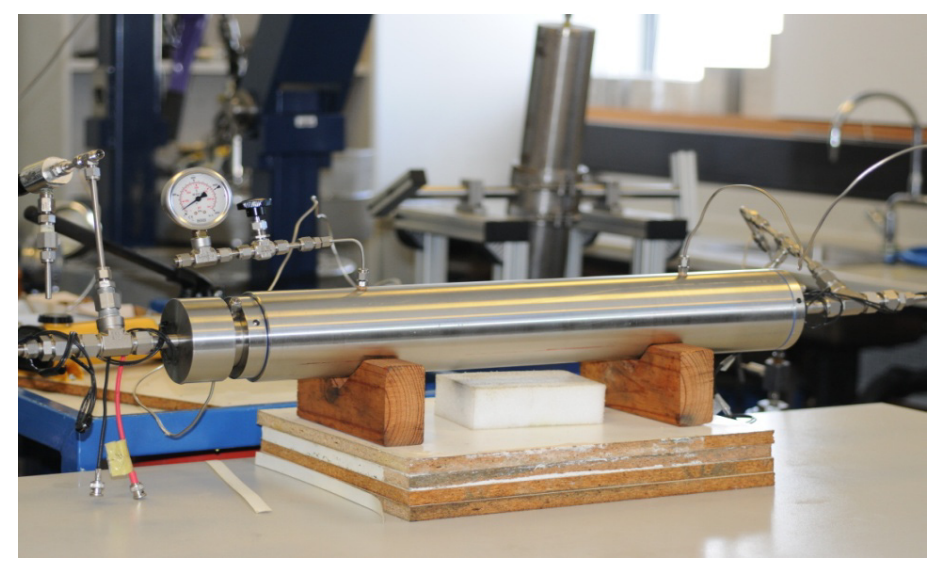

Figure 2: Hydrostatic core flooding setup.

\section{Challenges in lab experiments}

This section details the challenges experienced during experimental procedures and also details literature reviews of other problems evident when test permeability through porous media.

\subsection{Effect of saturating and injecting fluid}

The saturating fluid selection for any flooding test is a critical task. The saturating fluid should be inert to avoid any chemical reaction in the porous 
media and as a result alteration in permeability measurements. These chemical reactions can change the characteristics of the porous media in either way of increase or decrease in permeability. A suitable inert fluid when testing in lab condition would be deionised water with few present of $\mathrm{KCl}$ salt.

Choosing a saturating fluid is critical when performing tests on porous media and permeability. The saturating fluid should be inert to avoid any chemical reaction in the porous media and as a result alteration in permeability. When a reactive fluid flows through a porous media, it may dissolve some solid materials. The dissolved minerals species are in ionic form and can form precipitates with other ions already present in the fluid or can be transported downstream to form precipitates with other dissolved ions (Civan and Chang [4]). A suitable inert fluid when testing in lab condition would be deionised water to eliminate any chance of precipitates forming. However, in cores with clays present, water would not be a suitable choice. Clay minerals have high surface area to volume ratio and have a readiness to react with various invading fluids. Because of this property, clay minerals have become an ongoing problem for many experiments. When clay minerals contact some fluids, they tend to swell, disperse, or they are released from the pore wall if the salinity and $\mathrm{pH}$ of the fluids are unfavourable for the stability of clays (Civan and Chang [4]). This results in a reduction of porosity and permeability in the porous media.

If the injecting fluid is different from the saturating fluid, it is important to consider that if the injecting pressure during velocity test increases more than the maximum pressure of injecting fluid to displace the saturating fluid. If so, the permeability increases as the saturation of injecting fluid increases. Similar to the saturating fluid, the injecting fluid should be inert to preserve the permeability of the porous media.

\subsection{Effect of saturating pressure}

The saturation pressure is the pressure provided by the system to saturate the porous media with a fluid. As the pressure increases, the space provided for saturation increases and as a result the permeability increases. Consideration in this aspect should be noted to have the saturation pressure as the maximum limit for the inlet pressure during the core flood experiment. Using a pressure more than the maximum would result in an increase in the fluid saturation and therefore permeability. This can be potentially misunderstood as a failure within the porous media. Figure 3 demonstrates this aspect.

As shown in this figure, the permeability increases by increments of flow rate until it reaches a plateau at flow rates of $0.5 \mathrm{cc} / \mathrm{min}$. The permeability is enhanced because the injecting fluid saturation is increased due to an increase in mean pressure; however, the saturation remains stabilised after a certain mean pressure. This results in a constant permeability measurement after $0.5 \mathrm{cc} / \mathrm{min}$ flow rate. 


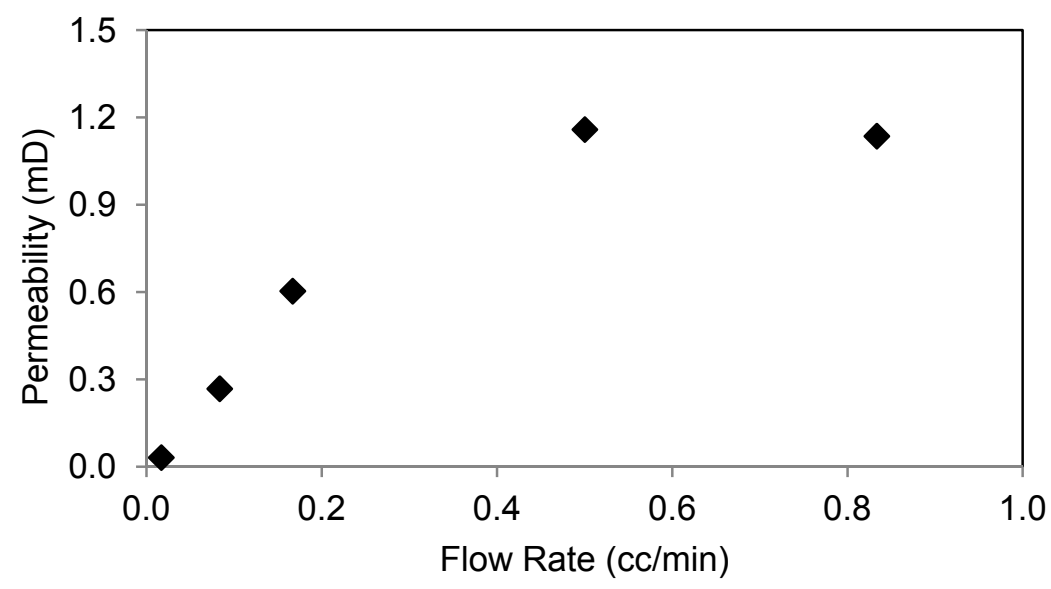

Figure 3: Permeability alteration due to exceeding the saturation pressure.

\subsection{The effect of overburden pressure}

During the core flood experiment, the overburden pressure is set to avoid the cross flow across the core sample. Since overburden pressure affects the core permeability, the difference between the overburden pressure and the mean pressure of flow should be kept constant to minimize the effective stress on the pore space.

An experiment performed by Ali et al. [5] found that on sandstone samples tested with increasing overburden pressure, the horizontal and vertical permeability decreased. A schematic of the results seen are shown in Figure 4.

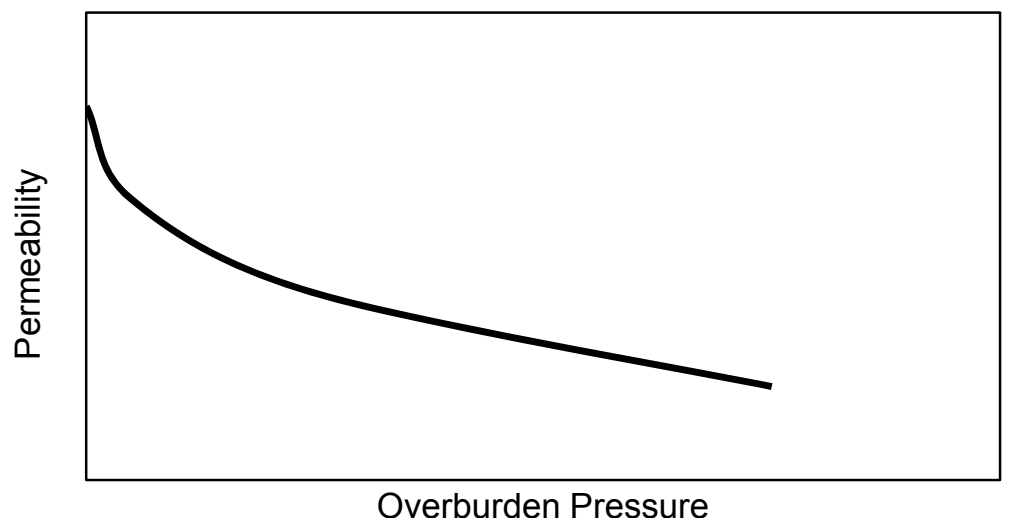

Figure 4: $\quad$ Effect of permeability with overburden pressure. 
Another effect of the overburden pressure is its preventing role in failure of the porous media. As the inlet pressure increases, the mean pressure lifts up. The mean pressure is basically pore pressure in the porous media, which increases boosting the possibility of the failure. This failure in the porous media affects the permeability drastically. Since the objective is to investigate the turbulent flow and as this regime occurs at high flow rates and mean pressure, it is important to set the overburden pressure to a reasonable value to supply enough confidence to prevent failure.

During the testing phase of the experiment, which is shown in Figure 5, the permeability did not obey the normal trend, i.e. decreasing with increments of flow rates. It is legitimated that rock failure occurred in the rock sample due to an increase in the mean pressure before turbulent regime initiated. The core sample underwent permanent changes and therefore the results are not representative of the initial core sample.

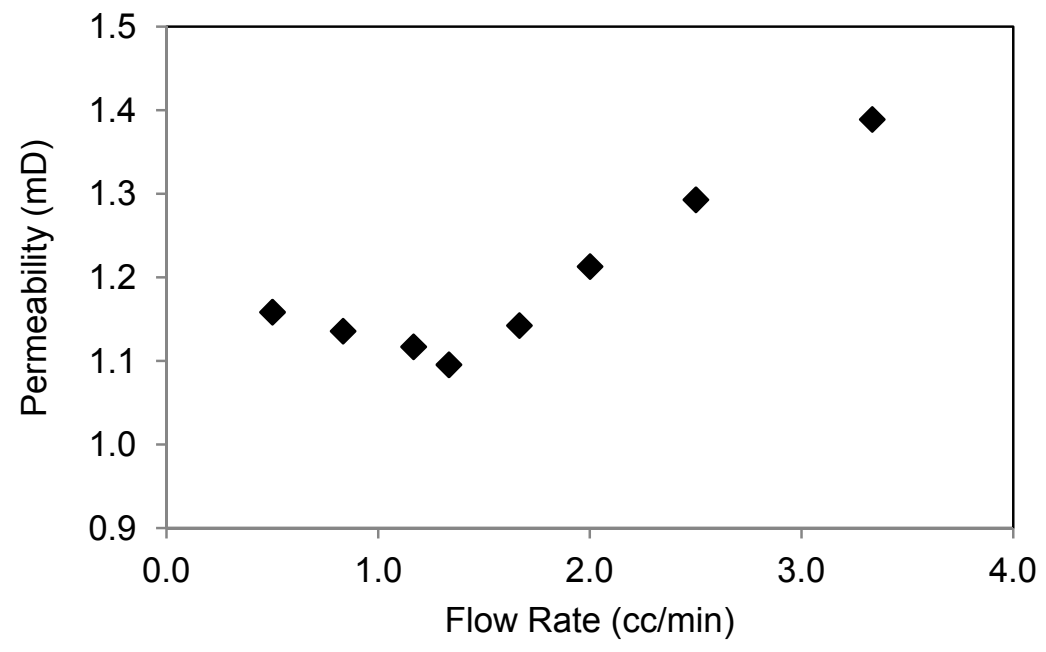

Figure 5: Permeability alteration due to failure in the core sample.

\section{Test profile}

A test profile is suggested here that can be used for either finding the boundary of the laminar-turbulent regimes or characterising the turbulent regime. The basis of this procedure is to differentiate between permanent permeability alterations and temporarily or flow rate dependent permeability reduction. Turbulent regime is considered as the temporary source of permeability reduction, as there is additional pressure loss in the porous media within this regime. This additional pressure loss is vanished once the flow rate decreases and flow pattern returns to laminar flow. 
An example of this discrimination is introduced here through a linear hydrostatic core flooding experiment. The base flow rate in this experiment was $0.5 \mathrm{cc} / \mathrm{min}$. This was placed in the laminar regime, as by decreasing the flow rate to $0.25 \mathrm{cc} / \mathrm{min}$ flow characteristics does not change. The permeability value within the laminar flow was measured as $0.46 \mathrm{md}$.

As shown in Figure 6, the permeability started to decrease at flow rate of 1 $\mathrm{cc} / \mathrm{min}$ and returned to its initial value once the flow rate decreased to $0.5 \mathrm{cc} / \mathrm{min}$. Therefore, it is legitimated to consider respective flow rate as the onset of turbulent regime. At higher increments of flow rate, the permeability decreases which follows the trend introduced by Forchheimer in 1901 (Barree and Conway [2]). Finally, the last experiment at the basis flow rate showed that the porous media had not been changed during the tests and the permeability values in turbulent regimes can be used for characteristics of this regime.

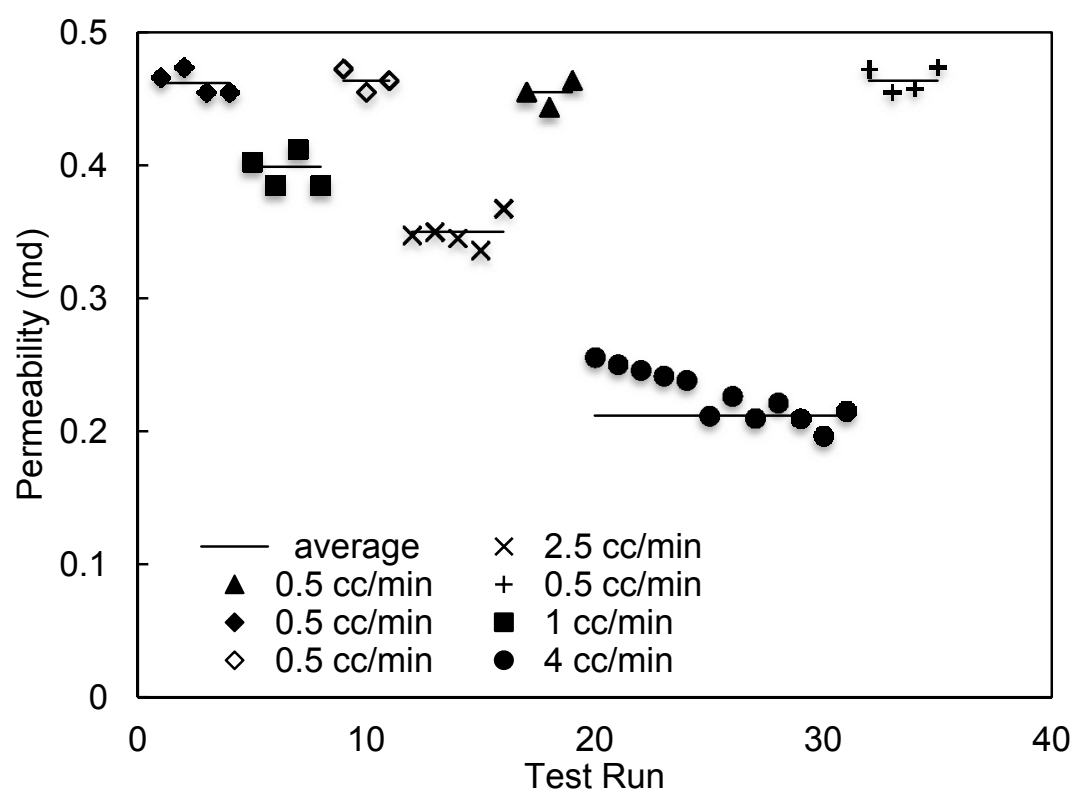

Figure 6: Permeability evolution through different flow rates.

\section{Conclusions}

The discussion in this paper indicated that testing permeability in laboratory conditions is tedious and requires accurate measurement and procedures. As explained in this paper, during the testing phase, many complications were found to disrupt the calculated permeability. The most common challenges that cause a fluctuation in the permeability are the saturation pressure and injecting pressure. It was discussed if the sample is not correctly saturated; the increase in flow rate across the cylinder increases the saturating capability of the sample, and thus 
increases permeability. The other challenge of laboratory measurements of permeability was mentioned to be the saturating and injecting fluid, in terms of chemical reactions within the sample and the effect of overburden pressure and fracture. Clay swelling and excessive pressure effects demonstrate a decrease in permeability and fracture within the sample result in an increase in permeability. All these factors make it challenging when trying to experiment on the permeability of a sample in the laminar and turbulent regime.

Finally, it was discussed that a proper test profile is required to differentiate between the temporarily and permanent permeability alteration for determination of the boundary of laminar and turbulent regimes. Any permeability measurement should be visited by this filter, to evaluate how the results are representative of the porous media response in both regimes of flow. The test procedure proposed here can be applied during flooding experiments for either determining the beginning and also characteristics of turbulent regime.

\section{References}

[1] Tarek, A., and McKinney, P.D., Advanced Reservoir Engineering. Burlington, Massachusetts: Elsevier, 2005.

[2] Barree, D. R. and, Conway, M.W., Beyond Beta Factors: A Complete Model for Darcy, Forchheimer, and Trans-Forchheimer Flow in Porous Media, SPE Annual Technical Conference and Exhibition. Houston, Texas: Society of Petroleum Engineer, pp. 1-8, 2004.

[3] Macini, P., Mesini, E., and Viola, R., Laboratory measurements of nonDarcy flow coefficients in natural and artificial unconsolidated porous media, Journal of Petroleum Science and Engineering, p.p. 365-374, 2011.

[4] Civan, F., and Chang, F., Modeling of Formation Damage Due to Physical and Chemical Interations Between Fluids and Reservoir Rocks, SPE Annual Technical Conference and Exhibition. Dallas: Society of Petroleum Engineers, p.p. 1-15, 1991.

[5] Ali, H.S., Al-Marhoun, M.A., Abu-Khamsin, S.A., and Celik, M.S., The Effect of Overburden Pressure on Relative Permeability, Middle East Oil Show. Bahrain: Society of Petroleum Engineers, p.p. 1-6, 1987. 